\title{
Algorithmes polynomiaux pour la recherche d'un chemin optimal dans une scène planifiée
}

Revue française d'automatique, d'informatique et de recherche opérationnelle. Recherche opérationnelle, tome 16, $\mathrm{n}^{\circ} 2$ (1982), p. 131-154.

$<$ http://www.numdam.org/item?id=RO_1982_16_2_131_0>

(C) AFCET, 1982, tous droits réservés.

L'accès aux archives de la revue « Revue française d'automatique, d'informatique et de recherche opérationnelle. Recherche opérationnelle » implique l'accord avec les conditions générales d'utilisation (http://www.numdam.org/ legal.php). Toute utilisation commerciale ou impression systématique est constitutive d'une infraction pénale. Toute copie ou impression de ce fichier doit contenir la présente mention de copyright.

\section{Numdam}

Article numérisé dans le cadre du programme

Numérisation de documents anciens mathématiques

http://www.numdam.org/ 


\title{
ALGORITHMES POLYNOMIAUX POUR LA RECHERCHE D'UN CHEMIN OPTIMAL DANS UNE SCĖNE PLANIFÉE ( $\left.{ }^{*}\right)$
}

\author{
par Y. Kergall $\left(^{1}\right)$
}

\begin{abstract}
Résumé. - Nous proposons des algorithmes polynomiaux pour résoudre le problème de la recherche d'un chemin de coût minimal dans une scène planifiée lorsqu'on ne considère qu'un seul critère, lorsqu'on considère une hiérarchie de critères ou encore le problème muticritère.

Mots-clés : algorithme, chemin optimal, graphe, robotique.

Abstract. - We propose polynomial algorithms for solving the problem of the search of a minimum cost path in a planified area. We may consider either only one criterion or a hierarchy of criteria, or the problem with several criteria.
\end{abstract}

Keywords: Algorithms, optimal path, graph, robotics.

\section{INTRODUCTION}

Un article récent [1] présenté au 2nd Congrès de Reconnaissance des formes et Intelligence artificielle de Toulouse (septembre 1979), propose un algorithme de type " énumératif » pour résoudre le problème de la recherche d'un chemin optimal par le biais d'une hiérarchie de critères, dans une scène planifiée.

Il existe de nombreux algorithmes polynomiaux classiques $\left({ }^{2}\right)$ en théorie des graphes et recherche opérationnelle pour résoudre le problème du plus court chemin : Ford, Ford amélioré, Bellman, Moore-Dijkstra ou algorithmes matriciels. Tous ces algorithmes opèrent sur des graphes valués. Or, dès que nous travaillons avec le critère de changement de direction, il n'est plus possible de valuer le graphe représentatif de la relation de voisinage entre les cases. Nous verrons de plus sur un exemple au paragraphe 6 que les chemins construits ici ne vérifient plus le théorème d'optimalité de Bellman, très général en recherche opérationnelle, puisque le coût d'un chemin né dépend pas seulement des arêtes parcourues ou des sommets traversés mais de la façon dont se suivent les arêtes sur le chemin.

(*) Reçu juin 1981 .

( $\left.{ }^{1}\right)$ Université de Tours, Laboratoire d'Informatique appliquée, parc Grandmont, 37200 Tours et depuis le $1 / 10 / 81$ : Centre universitaire, informatique, 33, rue Louis-Pasteur, 84000 Avignon.

$\left({ }^{2}\right)$ Pour toutes les notions classiques sur graphes et algorithmes (voir Gondran et Minoux [2]). 
Néanmoins, si ces algorithmes classiques ne peuvent être directement utilisés, nous proposons de les adapter à notre problème en leur conservant leur caractère polynomial.

En effet tous ces algorithmes reviennent à décomposer un graphe en niveaux et nous proposons ici de décomposer la scène en niveaux à partir de la case départ, sans construire de graphe représentatif de la scène qui est une perte de temps et de place mémoire.

L'utilisation de procédures énumératives [2], [6], ne nous semble pas s'imposer lorsque la scène est entièrement connue ou qu'elle est de taille modeste (jusqu'à $100 \times 100$ ) ce qui est bien souvent suffisant dans les applications.

Dans d'autres cas relevant de l'intelligence artificielle (univers partiellement inconnu au départ et appréhendé à l'aide de capteurs de différents types, univers de très grande dimension), on aura recours à d'autres algorithmes $[3,4,5,8]$, basés sur l'utilisation d'heuristiques.

Lorsque dans un problème interviennent un grand nombre de variables, trois types de méthodes permettent de réduire ce nombre de variables :

(1) réduire la taille de la structure de données (généralement une arborescence) qui est la représentation interne du problème : méthode $\alpha-\beta$, Graph Traverser de Doran et Michie [9], Flow-down method [10], etc.;

(2) partitionner le problème en $k$ sous-problèmes [6];

(3) regrouper les variables en méta-variables ce que nous faisons ici en définissant et construisant des "blocs " horizontaux ou verticaux, notion qui nous semble assez bien représenter le travail effectué par un opérateur humain confronté à un labyrinthe lorsqu'apparait le critère de changement de direction.

Cette notion permet bien entendu de réduire le nombre de variables de départ (les cases) mais sans trop compliquer les relations sur les " méta-variables " obtenues. En particulier la notion de pavé maximal (au sens de l'inclusion) comme rectangle formé de cases autorisés, notion analogue à celle de cellule dans [4] semble mal adaptée ici vu la difficulté à retrouver la suite des cases à emprunter une fois la suite des pavés trouvée.

Remarquons enfin que si dans [1] l'exploration de la scène en profondeur d'abord conduit à essayer d'améliorer pas à pas un chemin initial non optimal mais néanmoins le meilleur possible (à l'aide d'une heuristique à rapprocher de celle utilisée par Hart [3] dans l'algorithme classique $A^{*}$ ), les parcours en largeur d'abord utilisés ici permettent de compléter un début de chemin optimal.

Après avoir défini le problème, présenté l'article [1] et proposé des simplifications préliminaires sur la scène, nous proposons deux algorithmes pour le problème de hiérarchie de critères et le problème multicritère. Leur complexité 
est ensuite étudiée et nous donnons quelques temps d'exécution en fonction de divers paramètres pour des exemples tirés au hasard. Les programmes sont en annexe.

\section{POSITION DU PROBLEME}

\section{Définition d'une scène planifiée}

Nous appelons scène planifiée, une aire de déplacement rectangulaire de $M$ cases sur $N$ cases, que l'on pourrait représenter par une matrice de type $M \times N$. Nous avons préféré, pour des raisons d'efficacité de programmation, associer à chaque case $(i, j)$ de la scène, avec $1 \leqq i \leqq M$ et $1 \leqq j \leqq N$, une valeur $x=(i-1) N+j$. Ainsi on définit la scène à l'aide d'un vecteur, noté LAB, de la façon suivante : pour $1 \leqq x \leqq M N: \operatorname{LAB}(x)=0$ si la case $x$ est interdite (obstacle); $\operatorname{LAB}(x)=1$ si la case $x$ est autorisée.

Chaque case, non située sur un bord de la scène, possède 4 cases voisines. Un chemin est un ensemble de cases autorisées voisines. Le problème est la recherche d'un chemin « optimal » entre une case de départ notée $D$ et une case d'arrivée notée $A$.

\section{Critères d'optimalité}

Nous nous sommes intéressés aux deux critères suivants : $\mathrm{C} 1$, nombre de cases traversées; $\mathrm{C} 2$, nombre de changements de direction.

Problème 1: On considère les deux critères séparément et on recherche un chemin optimal pour $\mathrm{C} 1$ ou $\mathrm{C} 2$ c'est-à-dire comprenant un nombre minimal de cases traversées ou un nombre minimal de changements de direction.

Problème 2: Hiérarchie de critères : un chemin optimal sera parmi les chemins optimaux pour $\mathrm{C} 2$, un chemin optimal pour $\mathrm{C} 1$ [problème considéré dans (1)].

Problème 3 : Plus généralement soit $b$ le coût représenté par le fait de traverser une case, $a$ le coût d'un changement de direction. Soit $n_{1}$ le nombre de changements de direction et $n_{2}$ le nombre de cases traversées pour un chemin allant de $D$ à une case $x$. La valuation de cette case $x$ s'écrit $v(x)=n_{1} a+n_{2} b$.

On se propose de chercher $v^{*}(x)=\min v(x)$ et de déterminer un chemin correspondant à cet optimum. 


\section{METTHODE DELANNOY-LEROI-BOURTON [1]}

Cette méthode comprend 3 parties :

(1) réduction du nombre de cases autorisées;

(2) recherche d'un premier chemin entre $D$ et $A$;

(3) amélioration de ce chemin.

\subsection{Réduction du nombre de cases autorisées}

En notant $d^{+}$le nombre de cases voisines d'une case donnée, on élimine au départ toutes les cases - sauf $A$ et $D-$ telles que $d^{+}=1$, et que l'on appelle « culde-sac » dans la suite. On améliorera cette réduction au paragraphe 3.

\subsection{Recherche d'un premier chemin entre $D$ et $A$}

Ce chemin va servir d'initialisation à cette méthode qui est une méthode de type "énumératif». Ce premier chemin est construit avec l'heuristique suivante : en cours de construction du chemin, la suivante d'une case est sa voisine qui minimise la distance euclidienne à $A$.

\subsection{Amélioration de ce chemin}

Pour cela on essaie d'améliorer tout sous -chemin compris entre 2 sommets vérifiant $d^{+}>2$ (points de branchement). On ne sera assuré de l'optimalité que si l'on énumère tous les sous-chemins possibles. On connait les limites de telles procédures, même lorsqu'on leur applique certaines règles réduisant le nombre d'opérations envisagées (6). La règle de préclusion (sur les poids des chemins) est par exemple appliquée ici, qui abandonne tout début de chemin dont le poids atteint celui du meilleur chemin trouvé jusqu'alors.

\section{SIMPLIFICATIONS PRÉLIMINAIRES DE LA SCẺNE}

Les algorithmes présentés ici seront d'autant plus performants que la scène possède moins de cases autorisées, aussi va-t'on essayer de réduire a priori le nombre de cases permises. 
Exemple (fig. 1) :

Figure 1

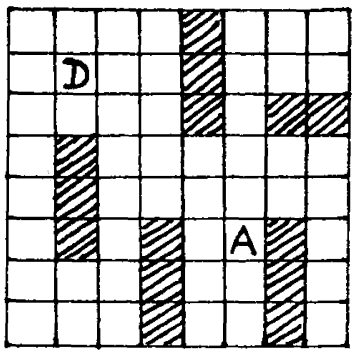

Figure 2

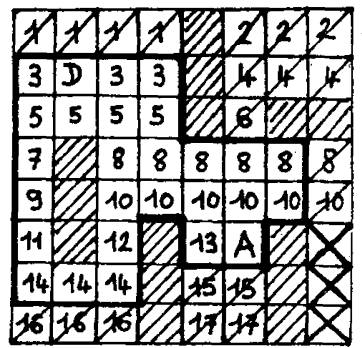

On élimine d'abord les portions en culs-de-sac (marquées X sur la figure 2); pour cela on peut, dans un premier passage, éliminer tous les culs-de-sac de la scène. Puis on rebalaye la scène en éliminant les nouveaux culs-de-sac, etc., jusqu'à ce que l'on ne puisse plus rien éliminer. Si $\lambda$ est la longueur maximale d'une portion en cul-de-sac, la complexité est en $O[(\lambda+1) M N]$.

Nous avons préféré prògrammer la méthode suivante, présentée ici sous une forme recursive :

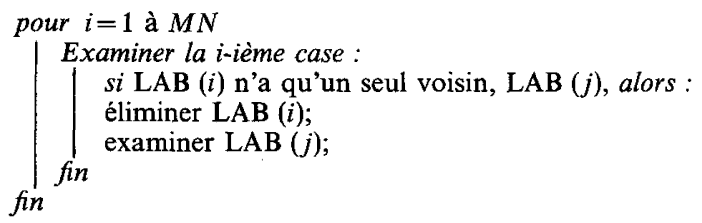

On génère un «backtrack » (retour en arrière) de profondeur maximale $\lambda$. On examine ici au maximum $M N+\Sigma \lambda_{k}$ cases, où $\Sigma \lambda_{k}$ représente le nombre total des cases appartenant aux portions en culs-de-sac, d'où une complexité en $O(M N)$.

On définit ensuite des blocs horizontaux comme des ensembles maximaux (au sens de l'inclusion) de cases autorisées voisines situées sur une même ligne. Sur la figure 2 on a affecté du même numéro les cases appartenant au même bloc horizontal.

Définissons le graphe $G_{H}$ de voisinage des blocs horizontaux par : soit 2 blocs horizontaux $i$ et $j$ alors $(i, j) \in G_{H} \Leftrightarrow$ il existe au moins une case du bloc $i$ et une case $\mathrm{du}$ bloc $j$ qui sont voisines. On supprimera de la scène toutes les cases appartenant à un bloc horizontal qui est une feuille de $G_{H}$ et qui ne contient pas $D$ ou $A$ (une feuille supprimée est notée $\bullet \#$ sur la figure 3). Ce graphe $G_{H}$ pourrait être réutilisé pour trouver un chemin présentant un nombre minimal de changements de direction ( $\$ 4.2$ ). En définissant les blocs verticaux et $G_{V}$ graphe de voisinage des blocs verticaux, on supprime aussi les feuilles de $G_{V}$. 


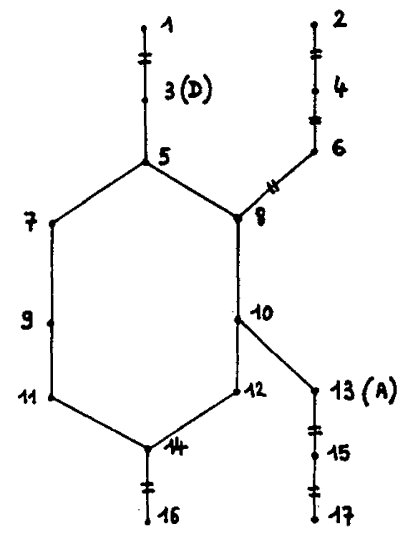

Figure 3

Pour l'exemple ci-dessus, les cases marquées $\square$ sur la figure 2 sont éliminées. Cette simplification préliminaire permet de supprimer toutes les cases situées, récursivement, sur les " bords " de la scène, en particulier des cases ayant au départ un nombre quelconque de voisins.

\section{PROBLÈME 1}

\subsection{Nombre minimal de cases traversées}

Nous notons ici $l_{1}(x)$, la longueur c'est-à-dire le nombre de cases traversées par un chemin allant de $D$ à la case $x$. La détermination de $l_{1}^{*}(A)$ qui est la longueur mınımale d'un chemin de $D$ à $A$ ainsi que le chemin correspondant est très simple puisqu'il suffit de décomposer le scène en niveaux $N_{i}$ à partir de $D$ en posant :

$$
N_{0}=\{D\},
$$

$N_{i}=\left\{\right.$ ensemble des cases $x$ voisines d'une case de $N_{i-1}$

et non encore atteintes $\}$.

Il est évident que l'on peut écrire :

$$
N_{i}=\left\{\operatorname{cases} x / l_{1}^{*}(x)=i\right\} .
$$

On arête la décomposition en niveaux dès qu'il existe $j$ tel que $N_{j}=\emptyset$ (dans ce cas le problème n'a pas de solution) ou dès que $A$ est atteinte; dans ce cas en notant $\lambda=l_{1}^{*}(A)$, un chemin solution est une suite de cases voisines partant de $A$ et de valuations $\lambda, \lambda_{-1}, \lambda_{-2}, \ldots, 2,1,0$. 
Remarque : Il est clair que ce que nous faisons ici est la décomposition en niveaux du graphe $G_{R}$ représentatif de la scène. Ce graphe pourrait être défini par :

$$
G_{R}=(X, \Gamma)
$$

$X$ : ensemble des cases autorisées.

$\Gamma$ : pour deux cases $i$ et $j,(i, j) \in \Gamma \Leftrightarrow i$ et $j$ sont voisines. Il est bien sûr inutile de construire explicitement $G_{R}$ ni même de se le donner sous la forme d'une case et de la liste de ses voisines (1) qui occupe encore un nombre de mémoires égal à 4 fois le nombre de cases libres ce qui peut être considérable sur une scène $100 \times 100$. En effet nous regarderons en cours d'algorithme quelles sont les cases libres voisines d'une case rencontrée, puisque chaque case ne sera considérée qu'une seule fois, sauf au paragraphe 6 .

Exemple: Sur la figure 4.

\subsection{Nombre minimal de changements de direction}

Nous notons $l_{2}(x)$ la longueur, c'est-à-dire le nombre de changements de direction pour un chemin allant de $D$ à $x$. Nous cherchons $l_{2}^{*}(A)$ et le chemin correspondant. Nous proposons une nouvelle décomposition en niveaux en notant :

$N_{0}=D \cup\{$ cases que l'on peut atteindre

sans changer de direction (directement) à partir de $D\}$.

$N_{i}=\{$ cases non atteintes

et que l'on peut atteindre directement à partir de $\left.N_{i-1}\right\}$.

On s'arrête dès qu'il existe $j$ tel que $N_{j}=\emptyset$ ou dès que l'on atteint $A$. Si $A$ appartient à $N_{k}$ alors $l_{2}^{*}(A)=k$.

En effet si $A$ appartient à $N_{k}$ alors $l_{2}(A)=k$.

De plus $k=\min l_{2}(A)=l_{2}^{*}(A)$ puisqu'on s'arrête dès que l'on atteint $A$, c'està-dire qu'il n'existe pas de niveau $N_{l}, l<k$, et $\mathrm{A} \in N_{l}$.

Pour retrouver un chemin minimal, il suffit au cours de l'algorithme de noter chaque fois que l'on atteint une case $x$ de $N_{i}$, à partir de quelle case $y$ de $N_{i-1}$ on a pu atteindre $x$. On posera $\operatorname{PRED}(x)=y$ qui nous donnera ainsi la liste des prédecesseurs de chaque sommet. En d'autres termes PRED contient la liste des sommets où l'on a changé de direction entre $D$ et $A$; entre 2 de ces sommets, le chemin en ligne droite est évident à reconstituer. 


\section{Exemple :}

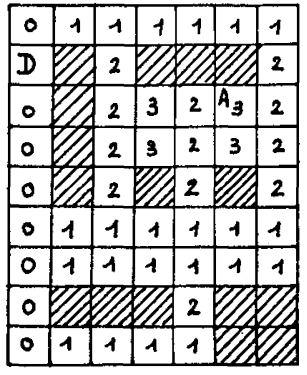

$t_{2}^{*}(\mathrm{~A})=3$

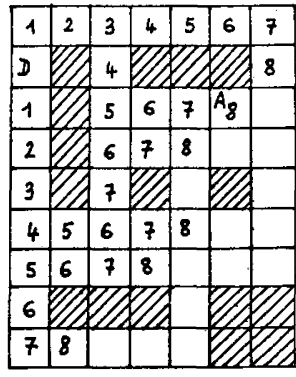

$t_{1}^{*}(\mathrm{~A})=8$

Figure 4

\subsection{Utilisation de $G_{H}(\S 3, f i g .3)$}

Dans le graphe $G_{H}$ nous avons représenté les blocs horizontaux et les liaisons entre eux. Ces arêtes entre 2 blocs horizontaux représentent des liaisons verticales que l'on peut songer à prolonger de façon à relier tout bloc horizontal $i$ à tout bloc horizontal $j$ s'il existe un bloc vertical entre $i$ et $j$ (fermeture transitive de la relation de voisinage entre 2 blocs horizontaux)

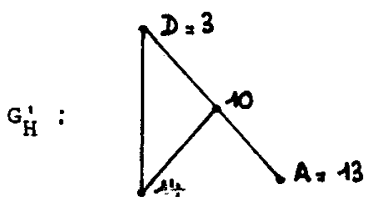

On obtient un graphe $G_{H}^{\prime}$ : A tout chemin $x_{0} x_{1} \ldots x_{n}$ de $G_{H}^{\prime}\left(D \in x_{0}, A \in x_{n}\right)$ on fait correspondre simplement un chemin dans la scène puisque l'on se déplace horizontalement dans tout bloc $x_{i}$ et verticalement entre tout couple $\left(x_{i}, x_{i+1}\right)$, $0 \leqq i \leqq n-1$. Le nombre de changements de direction est :

$$
l_{2}(A)=2(n-1)+\varepsilon+\varepsilon^{\prime},
$$

avec $\varepsilon=0$ si on peut atteindre directement, dans la scène, $x_{1}$ à partir de $D, 1$ sinon et $\varepsilon^{\prime}=0$ si on peut atteindre directement, dans la scène $x_{n-1}$ à partir de $A, 1$ sinon.

Pour le chemin $(3,10,13)$ de $G_{H}^{\prime}$ on trouve $l_{2}(A)=2+1+0=3$ et pour $(3,14$, $10,13), l_{2}(A)=4+1+0=5$.

On se ramène donc, à $\varepsilon+\varepsilon^{\prime}$ près, à la recherche d'un chemin comportant le moins d'arêtes dans un graphe très réduit.

R.A.I.R.O. Recherche opérationnelle/Operations Research 


\section{HIERARCHIE DE CRITÈRES DE CHOIX}

Disposant de 2 critères de choix, on commence par minimiser le critère « le plus coûteux ». Pour un robot, le changement de direction est l'un des critères les plus coûteux en temps donc en énergie. Nous résolvons alors le problème : parmi les chemins qui comportent un minimum de changements de direction, quels sont ceux qui traversent un nombre minimal de cases [problème étudié dans (1)].

Nous réappliquons l'algorithme vu en 4.2 , mais pour chaque niveau $N_{i}$, on associe de plus à toute case $x$ de $N_{i}$ sa valuation $l_{1}(x)$ obtenue de la façon suivante [où $d(x, y)$ représente le nombre de cases traversées pour aller (en ligne droite) de $x$ à $y]$ :

\subsection{Algorithme}

On rappelle (voir 4.2) que si une case $x$ est directement accessible à partir d'une case $y$, c'est que l'on peut aller de $y$ à $x$ sans changer de direction, c'est-àdire en suivant une horizontale ou une verticale de la scène :

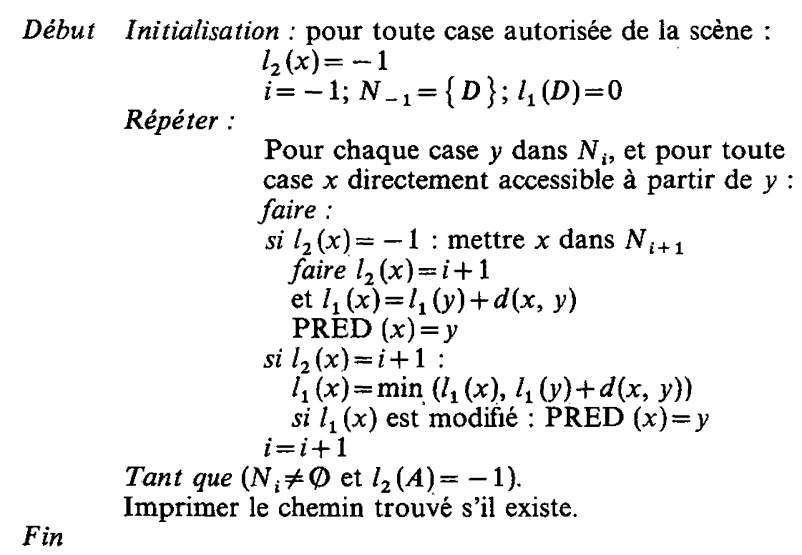

Preuve : Cet algorithme s'arrête si $N_{i}=\emptyset$ (il n'y a pas de solution) ou si $l_{2}(A) \neq-1$, le chemin étant alors facile à reconstituer à l'aide du tableau d'indice PRED qui permet de remonter jusqu'à $D$.

Pour tout $i \geqq 0$ on a $N_{i}=\left\{\right.$ cases $\left.x / l_{2}^{*}(x)=i\right\}$ puisque la construction est identique à celle du paragraphe 3.2 .

D'autre part il ne peut exister un chemin de $D$ à $A$ ayant $l_{2}^{*}(A)$ changements de direction et induisant une valeur $l_{1}(A)$ inférieure à celle trouvée par le chemin déduit du tableau PRED. En effet soit $\lambda=l_{2}^{*}(A)$. S'il existe deux prédecesseurs $y$ à $A$ dans $N_{\lambda-1}$ on mémorise dans PRED celui qui minimise $l_{1}(y)+d(A, y)$. On 
est donc assuré d'obtenir ainsi parmi les chemins possédant un nombre minimal de changements de direction, un chemin traversant un nombre minimal de cases de $D$ à $A$.

Notons que cet algorithme revient à construire pour chaque niveau $N_{i}$ et pour toute case $x$ de $N_{i}$, le bloc horizontal (ou vertical) maximal qui contient $x(\S 3)$.

Remarque : Si une case $x$ est atteinte horizontalement (resp. verticalement) alors il suffit de considérer à partir de $x$ les cases que l'on peut atteindre verticalement (resp. horizontalement). Cette simple remarque a permis de diviser les temps d'exécution pratiquement par 2, mais a conduit à rajouter un tableau de taille $M N$.

\section{Structure des données}

La scène est représentée en mémoire par un vecteur, $\operatorname{LAB}(x), 1 \leqq x \leqq M N$, de façon à réduire dans les programmes le nombre de calculs d'indices. Chaque case $x$ autorisée de la scène, contient en partie gauche $l_{2}(x)$ et $l_{1}(x)$ en partie droite. Cette association dans le même vecteur de 3 informations (définition de la scène, $l_{1}$ et $l_{2}$ ) a permis de réduire le temps d'exécution et la place mémoire utilisée.

Notons que cet algorithme revient à parcourir la scène en largeur d'abord (breadth-first-search) et que nous avons seulement besoin de 2 niveaux $N_{i}$ et $N_{i-1}$ simultanément en mémoire, lesquels sont gérés comme des files (si le parcours avait été de type profondeur d'abord, on aurait utilisé une pile [2], [11]).

\subsection{Complexité}

Nous notons $\tau$ le taux de cases interdites dans la scène. Nous avons choisi $0,10 \leqq \tau \leqq 0,35$, au-delà de 0,35 la scène ne présentant plus, en pratique, de chemin reliant le départ $D$ à l'arrivée $A$; on prend arbitrairement $D=1$ et $A=M . N$ pour avoir des ordres de grandeur maximale pour une scène de taille donnée $M \times N$.

Le nombre de cases autorisées $y$, considérées dans l'algorithme est inférieur à $(1-\tau) M N$. Le nombre "moyen " de cases interdites sur une ligne est $\tau N$ (espérance mathématique d'une loi binomiale de paramètre $\tau$ ). La distance " moyenne " entre 2 cases interdites est $N / \tau N=1 / \tau$ d'où le nombre moyen de cases directement accessibles, horizontalement ou verticalement (voir remarque du paragraphe 5) à partir d'une case y est $1 / \tau$. Ainsi le corps de la boucle, dans lequel le nombre d'instructions exécutées est indépendant de la taille de la scène, est traité $(1-\tau) M N .1 / \tau$ fois, d'où une complexité en $O(M N)$. Un seul niveau $N_{i-1}$, est nécessaire pour déterminer $N_{i}$. On aura donc seulement deux tableaux simultanément en mémoire avec la scène elle-même. La taille de ces tableaux, qui 
est égale au nombre de cases atteintes à un niveau, décroît avec $\tau$. Nous avons obtenu les valeurs moyennes suivantes pour $M=N=50$ :

TABLEAU I

\begin{tabular}{|c|c|c|c|c|c|c|c|}
\hline Taux....... & 0,10 & 0,15 & 0,20 & 0,25 & 0,30 & 0,35 & 0,40 \\
\hline Taille. . . . . . & 830 & 500 & 360 & 230 & 175 & 85 & 15 \\
\hline
\end{tabular}

La complexité spatiale totale a toujours été inférieure à $4 . M N$ dans tous les exemples traités.

Le tableau suivant donne les temps moyens d'exécution (en secondes) obtenus en fonction du taux et de la taille $=M=N$ de la scène (CII 10070 du C.I.C.R.C. d'Orléans-Tours).

TABLEAU II

\begin{tabular}{|c|c|c|c|c|c|c|}
\hline \multirow{2}{*}{ Taille } & \multicolumn{6}{|c|}{ Taux } \\
\hline & 0,10 & 0,15 & 0,20 & 0,25 & 0,30 & 0,35 \\
\hline $20 \times 20$ & 2 & 1 & 1 & 1 & 0 & 0 \\
\hline $30 \times 30$ & 2 & 2 & 2 & 2 & 2 & 1 \\
\hline $40 \times 40$ & 5 & 4 & 4 & 3 & 3 & - \\
\hline $50 \times 50$. & 7 & 6 & 6 & 5 & 4 & 3 \\
\hline $60 \times 60 \ldots$ & 11 & 9 & 7 & 6 & 6 & 6 \\
\hline $70 \times 70 \ldots$ & 13 & 9 & 7 & 6 & 6 & 4 \\
\hline $80 \times 80 \ldots$ & 15 & 12 & 9 & 7 & 7 & 6 \\
\hline $90 \times 90$. & 20 & 15 & 13 & 10 & 8 & - \\
\hline $100 \times 100 \ldots$ & 26 & 19 & 15 & 12 & - & - \\
\hline
\end{tabular}

\subsection{Commentaires}

Nous avons pu borner le nombre maximal de cases considérées à partir de chaque case autorisée, par une « constante », $1 / \tau$, indépendante de $M$ et $N$, d'où un temps en $O(M N)$. Dans le pire des cas (worst case), quand il n'y a aucun obstacle dans l'aire, $\tau$ est nulle et $1 / \tau=N$ c'est-à-dire que le nombre maximal de cases atteintes directement à partir d'une case donnée est borné par $N$ (ou $M$ ), et $\lim (1-\tau) M N .1 / \tau=M N^{2}$. La complexité maximale de l'algorithme HIERAR$\tau \rightarrow 0$

CHIE est en $O\left(M N^{2}\right)$.

\section{ALGORITHME MULTICRITÈRE}

Soit $n_{1}$ le nombre de changements de direction et $n_{2}$ le nombre de cases traversées pour un chemin allant de $D$ à une case $x, a$ le coût d'un changement de vol. $16, \mathrm{n}^{\circ} 2$, mai 1982 
direction et $b$ le coût dû au fait de traverser une case. La valuation associée par ce chemin à $x$ est alors $v(x)=n_{1} a+n_{2} b$. On notera $v^{*}(x)=\min v(x)$, calculé sur l'ensemble des chemins allant de $D$ à $x$. On cherche $v^{*}(A)$ et un chemin optimal correspondant.

Il est clair que les critères séparés ou une hiérarchie de critères ne construisent pas un chemin optimal pour $v$, comme le montre l'exemple ci-dessous dans lequel $a=2$ et $b=1$.

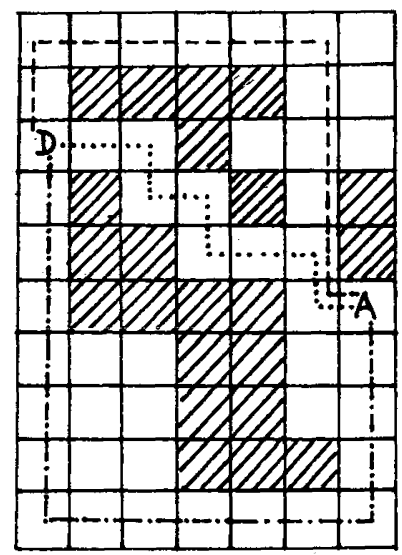

\begin{tabular}{|l|l|l|l|}
\hline & $n_{1}$ & $n_{2}$ & $v$ \\
\hline chemin $\ldots$ & 3 & 13 & 19 \\
\hline chemin ..... & $\overline{6}$ & 9 & 21 \\
\hline chemin $\ldots$ & 2 & 17 & 21 \\
\hline
\end{tabular}

Exemple traité en annexe

Pour traiter ce problème, nous construisons l'arborescence des chemins allant de $D$ à $A$. Soit $C H=\left\{D, x_{1}, x_{2}, \ldots, x_{i-1}, x_{i}\right\}$ un début de chemin allant de $D$ à la case $x_{i}$. Supposons que $v\left(x_{i}\right)=k$. Soit $y \notin C H$ une case voisine de $x_{i}$. Si $x_{i-1}, x_{i}, y$ est en ligne droite on pose $V(y)=k+b$ sinon on pose $v(y)=k+a+b$ puisqu'on a en plus un changement de direction pour passer de $x_{i}$ à $y$ en venant de $x_{i-1}$. On peut là aussi parler de décomposition en niveaux en posant : $y \in N_{k} \Leftrightarrow v(y)=k$. Une case $y$ peut apparaitre dans plusieurs niveaux contrairement à l'algorithme précédent.

Exemple $(a=3, b=1)$ : Considérons les 4 cases suivantes extraites d'une scène :

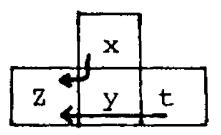

Supposons que l'on ait $v(x)=15$; alors $v(y)=16$ et $v(z)=20$. Supposons que l'on obtienne $v(t)=17$; alors $v(y)=18$. Cette valeur supérieure à $v(y)=16$ obtenue précédemment doit néanmoins être conservée puisqu'elle implique $v(z)=19$ meilleure que la valuation précédente de $v(z)$.

R.A.I.R.O. Recherche opérationnelle/Operations Research 
Ainsi, en posant $N_{0}=\{D\}$, la détermination de $N_{k}(k \geqq a+b)$ se fait à partir des niveaux $N_{k-b}$ et $N_{k-b-a}$. On doit donc conserver $(a+b+1)$ niveaux simultanément en mémoire, ce qui n'est pas une contrainte trop lourde car chaque niveau ne contient que peu de sommets et notablement moins qu'à l'algorithme précédent; en effet, dans l'algorithme précédent on pouvait atteindre directement à partir de chaque case $(\neq D)$ jusqu'à $\operatorname{Max}(M, N)-1$ cases, contre au plus 3 ici.

On essaie de préciser et réduire le nombre d'éléments de chaque niveau à l'aide des remarques et corollaires suivants.

Remarque 1 : Soit une case $y, y \in N_{k}$. Alors toute occurence de $y$ dans un niveau $N_{l}$ avec $l-k \geqq a$ est inutile.

En effet toute case $x$ obtenue à partir de l'occurence de $y$ dans $N_{l}$ possédera une valeur $v(x)$ supérieure ou égale à celle obtenue à partir de l'occurence de $y$ dans $N_{k}$ (règle classique de preclusion).

Remarque 2: Si 2 cases $x$ et $y$ se suivent dans le même ordre sur plusieurs chemins à partir de $D$, on peut ne garder que l'occurrence de $y$ qui présente la valuation $v(y)$ minimale.

En effet toute case $z$ obtenue à partir de l'une quelconque des occurrences de $y$, l'est de la même façon (c'est-à-dire avec ou sans changement de direction), et l'ordre sur les valuations de $y$ se conserve sur les valuations de tous les successeurs de $y$.

Cette remarque permet de fusionner plusieurs débuts de chemins, technique bien connue pour réduire la taille des arborescences (branch merging) [6].

COROLlaIre 1 : Toute case $x$ possédant $k$ cases voisines apparait au plus $k$ fois dans l'arborescence.

Les occurrences de $x$ doivent en effet d'après la remarque précédente être suivies de cases différentes.

COROLlaIRE 2 - Le nombre d'occurrences de sommets dans l'arborescence est inférieure à 4 fois le nombre de cases autorisées du labyrinthe.

L'emplacement maximal est donc linéaire en $M N$ et en fait très proche de $M N$ grâce à la suppression de toute occurrence d'une case dans un niveau $N_{l}$ dès que cette case apparait dans le niveau $N_{k}$, avec $l-k \geqq a$. Notons que ce nombre d'occurrences est d'autant plus proche de $M N$ que a est faible. Si $a=1$, pour toute case $x$ on peut ne garder qu'une occurrence, celle pour laquelle $v(x)$ est minimale.

Propositron : Soit $i=\min \left\{k / x \in N_{k}\right\} ;$ alors $i=v^{*}(x)$. Dire que $x$ appartient à $N_{k}$ c'est dire qu'il existe un chemin de $D \grave{a} x$ induisant une valuation $v(x)=k$. Donc $i=\min (v(x))=v^{*}(x)$. 


\section{Algorithme :}

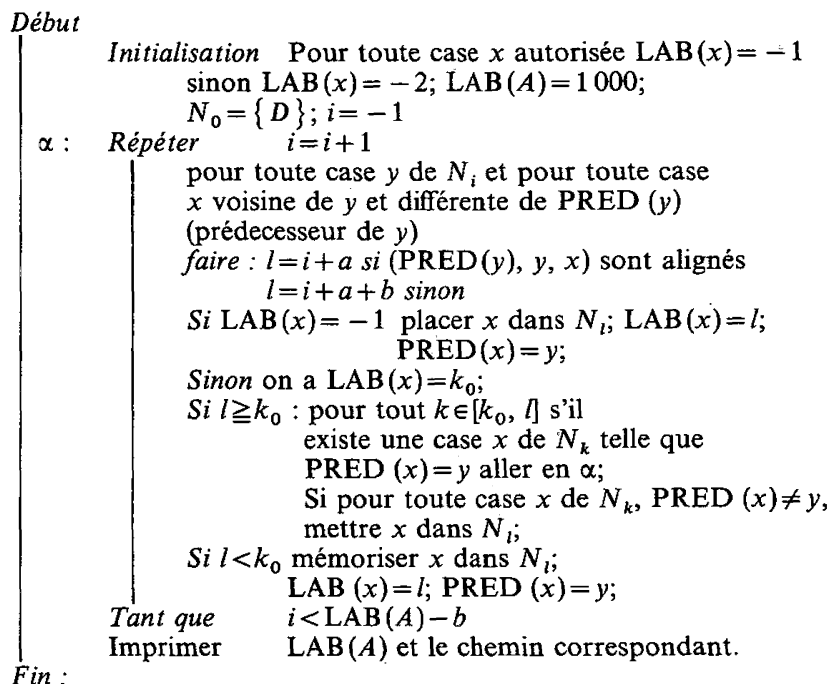

Remarque : Le problème n'a pas de solution s'il existe $a+b-1$ niveaux consécutifs vides, ce qui ne pose aucun problème au niveau de l'agorithme et du programme.

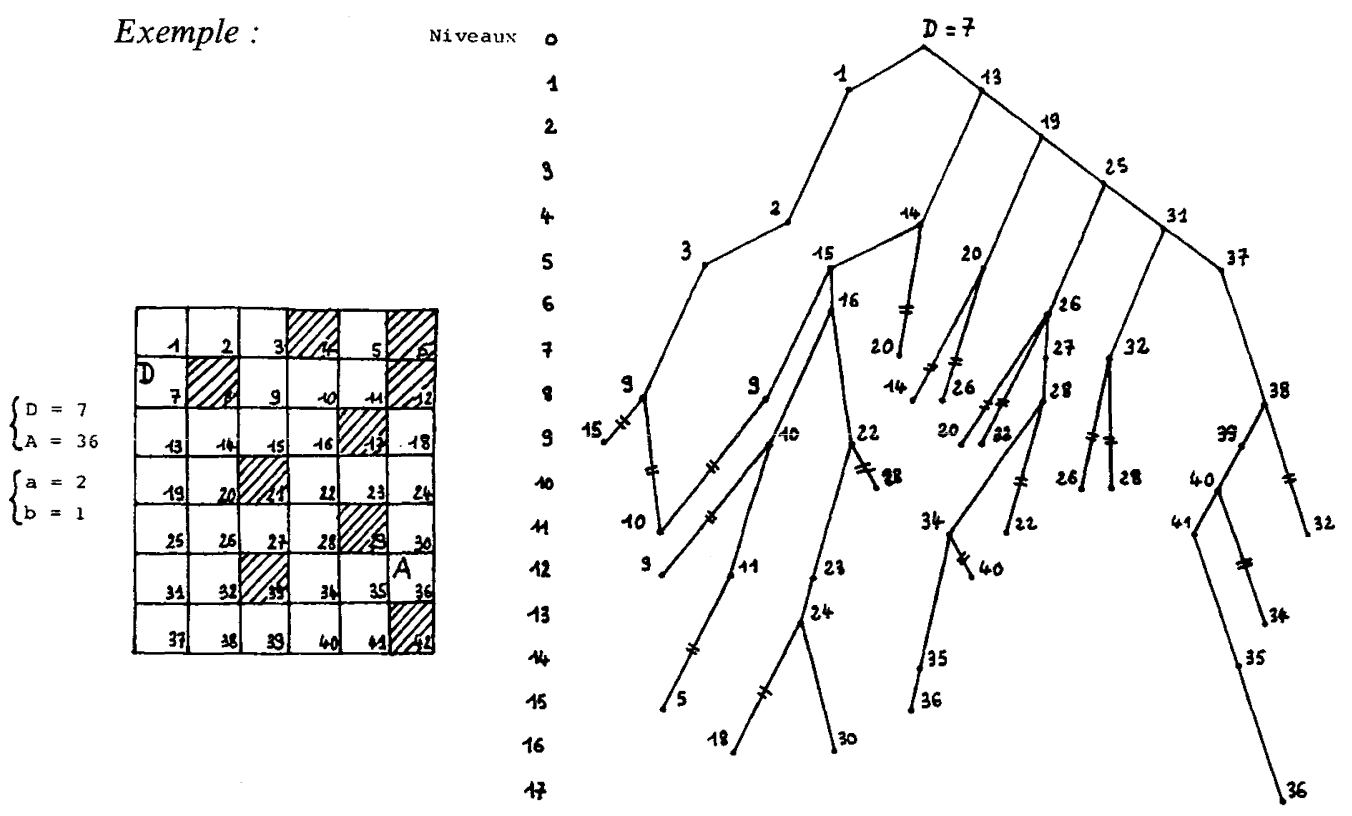

L'examen de $N_{14}$ implique $v^{*}(A)=14+b=15$.

Le chemin optimal est $\left\{7,13,19,25^{\circ}, 26,27,28,34,35,36\right\}$.

R.A.I.R.O. Recherche opérationnelle/Operations Research 
Même exemple avec $a=3, b=1$ :

Sur cet exemple, le principe d'optimalité de Bellmann n'est pas vérifié; le plus court chemin pour atteindre la case 35 (noté ......) n'est pas la portion entre $D$ et $35 \mathrm{du}$ plus court chemin pour atteindre la case 36 en passant par 35 (noté $----)$.

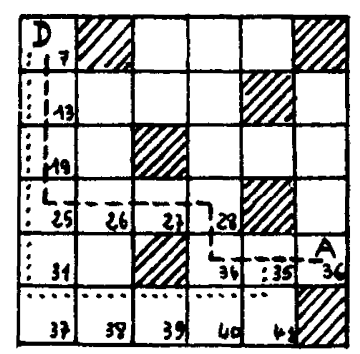

En effet le chemin en pointillé traverse 12 cases et présente trois changements de direction d'où une longueur de 21 entre $D$ et $A$ et une longueur de 17 entre $D$ et la case 35. Le chemin avec des tirés comporte 10 cases et 3 changements de direction soit une longueur de 19 entre $D$ et $A$, et une longueur de 18 entre $D$ et la case 35 .

\subsection{Complexité}

Chacune des $(1-\tau) M N$ cases autorisées est mémorisée au plus 4 fois dans l'arborescence et pour chacune de leurs 3 voisines on peut être amené à considérer au plus $a$ niveaux dont la taille a toujours été très largement bornée par $N$ dans nos exemples. Au pire, si $\tau=0$, le nombre maximal de cases atteintes à un niveau pourrait être de l'ordre de $N \sqrt{2}$ comme le montre la figure ci-contre dans laquelle $a=2, b=1$ et la valeur de chaque case représente le niveau dans lequel appartient chaque case, c'est-à-dire la longueur minimale pour un chemin allant de $D$ à cette case. Si $\tau$ croît, le nombre de niveaux augmente, et le nombre de cases par niveau décroît.

\begin{tabular}{|c|c|c|c|c|c|c|c|c|}
\hline D & 1 & $\begin{array}{ll}23 \\
2\end{array}$ & 4 & 5 & 16 & & 8 & \\
\hline 1 & 4 & 516 & 7 & 8 & 9 & 10 & & \\
\hline 2 & 5 & \begin{tabular}{l|l}
6 & 7
\end{tabular} & 8 & 19 & 10 & & & \\
\hline 3 & 6 & 78 & 19 & 10 & & & & \\
\hline 4 & \begin{tabular}{|l|l}
7 \\
\end{tabular} & \begin{tabular}{l|l}
89 \\
\end{tabular} & 19 & & & & & \\
\hline 5 & 8 & 911 & & & & & & \\
\hline 6 & 9 & 10] & & & & & & \\
\hline 7 & 10 & & & & & & & \\
\hline 8 & & & & & & & & \\
\hline
\end{tabular}

vol. $16, \mathrm{n}^{\circ} 2$, mai 1982 
En acceptant cette majoration en $O(N)$ on est conduit à un temps en $O\left(M N^{2}\right)$. La place mémoire utilisée n'a jamais excédé $2 M N$. Le tableau suivant donne les temps moyens d'exécution (en secondes) en fonction de $M \times N$. Il est à noter que ces temps sont pratiquement indépendants du taux $\tau$.

TABLEAU III

\begin{tabular}{|l|c|c|c|c|c|c|c|c|c|}
\hline Taille. & $20 \times 20$ & $30 \times 30$ & $40 \times 40$ & $50 \times 50$ & $60 \times 60$ & $70 \times 70$ & $80 \times 80$ & $90 \times 90$ & $100 \times 100$ \\
\hline Durée. & 1 & 2 & 4 & 5 & 6 & 7 & 8 & 11 & 13 \\
\hline
\end{tabular}

\subsection{Commentaires}

Il peut paraître paradoxal qu'un algorithme en $O\left(M N^{2}\right)$ donne certains temps moyens meilleurs que ceux d'un algorithme en $O(M N)$. En effet HIÉRARCHIE est plus lent que MULTICRITÈRE, mais seulement lorsque $\tau$ est inférieur à 0,20-0,25. On sait, d'autre part, que lorsque $\tau$ décroît, la rapidité de HIÉRARCHIE décroît aussi, puisqu'il y a, pour un nombre de niveaux constant, de plus en plus de cases à examiner, pour atteindre avec $\tau \sim 0$, le pire des cas. Avec MULTICRITĖRE, lorsque $\tau$ croît, il y a plus de niveaux à examiner mais moins de cases dans chaque niveau, et réciproquement lorsque $\tau$ décroît, ce qui explique l'indépendance des temps de MULTICRITÈRE par rapport à $\tau$. Dans l'algorithme Hiérarchie, nous avons pu borner le nombre maximal (notons le $\gamma$ ) de cases considérées à partir de chaque case autorisée par une "constante » $1 / \tau$, indépendante de $N$, d'où un temps en $O(M N)$. Au contraire, dans l'algorithme multicritère, $\gamma$ peut être au pire de l'ordre de $N$, mais en pratique lorsque $\tau$ est faible, $1 / \tau$ est supérieur à $\gamma$ ce qui explique la différence des temps. $O\left(M N^{2}\right)$ représente la complexité maximale pour les 2 algorithmes car $\lim 1 / \tau=N$ (c'est-à-dire que le nombre maximal de cases atteintes directement à $\tau \rightarrow 0$

partir d'une case donnée est aussi borné par $N$ dans l'algorithme multicritère, si $\tau=0$ (aucun obstacle dans l'aire). On connaît néanmoins les limites de la notation en $O(\quad)$ (voir par exemple [12]).

\section{PROGRAMMES}

A l'image de J. Arsac, nous pensons qu'il est impossible de dissocier algorithmique et programmation. Nous donnons en annexe les 2 programmes documentés. Nous avons essayé d'affiner au mieux la programmation : 3 versions successives ont été testées pour l'algorithme Hiérarchie dont bien sûr 
seule la plus rapide a été conservée. Notons par exemple que le fait d'associer dans la même mémoire $l_{1}$ et $l_{2}$ relatives à la même case $x$, a permis en supprimant une structure répétitive de diviser par 4 les temps d'exécution.

Notons enfin une heuristique utilisée dans l'algorithme multicritère pour retrouver plus rapidement le chemin de la case arrivée à la case départ à l'aide des valuations : une case $x$ étant trouvée, le prédécesseur essayé est d'abord la case située au-dessus de $x$ plutôt qu'au dessous, à gauche de $x$ plutôt qu'à droite, vues les dispositions des cases départ et arrivée.

Remarque : Des mots de 32 bits sont nécessaires pour Hiérarchie de façon à avoir 8 chiffres significatifs par mémoire ( 4 pour $l_{1}, 4$ pour $l_{2}$ ).

\section{CONCLUSION}

Nous avons traité ici les problèmes de parcours dans une aire comme des problèmes de décomposition d'un graphe en niveaux, la décomposition variant selon les critères d'optimalité choisis. Les algorithmes polynomiaux développés ici ont conduit à des temps très faibles pour des scènes dont nous avons fait varier la taille jusqu'à $100 \times 100$ (moins de 30 secondes sur un CII 10070). Ces algorithmes sont utilisables dès que la scène est entièrement déterminée ce qui peut se faire dans une première étape par des procédures énumératives (avec heuristiques) du domaine de l'intelligence artificielle. 


\section{ANNEXE}

Nous donnons en annexe les programmes correspondants à ces deux algorithmes ainsi que des exemples de scènes tirées au hasard avec les résultats fournis par ces algorithmes.

Hiérarchie de critères: Un exemple de résultats obtenus pour $M=N=50$ et un taux de 0,35 .

Si la valeur d'une case $=-2:$ case interdite; $=-1:$ case non atteinte; $=i \geqq 0$ : case atteinte en $i$ changements de direction à partir de $D$.

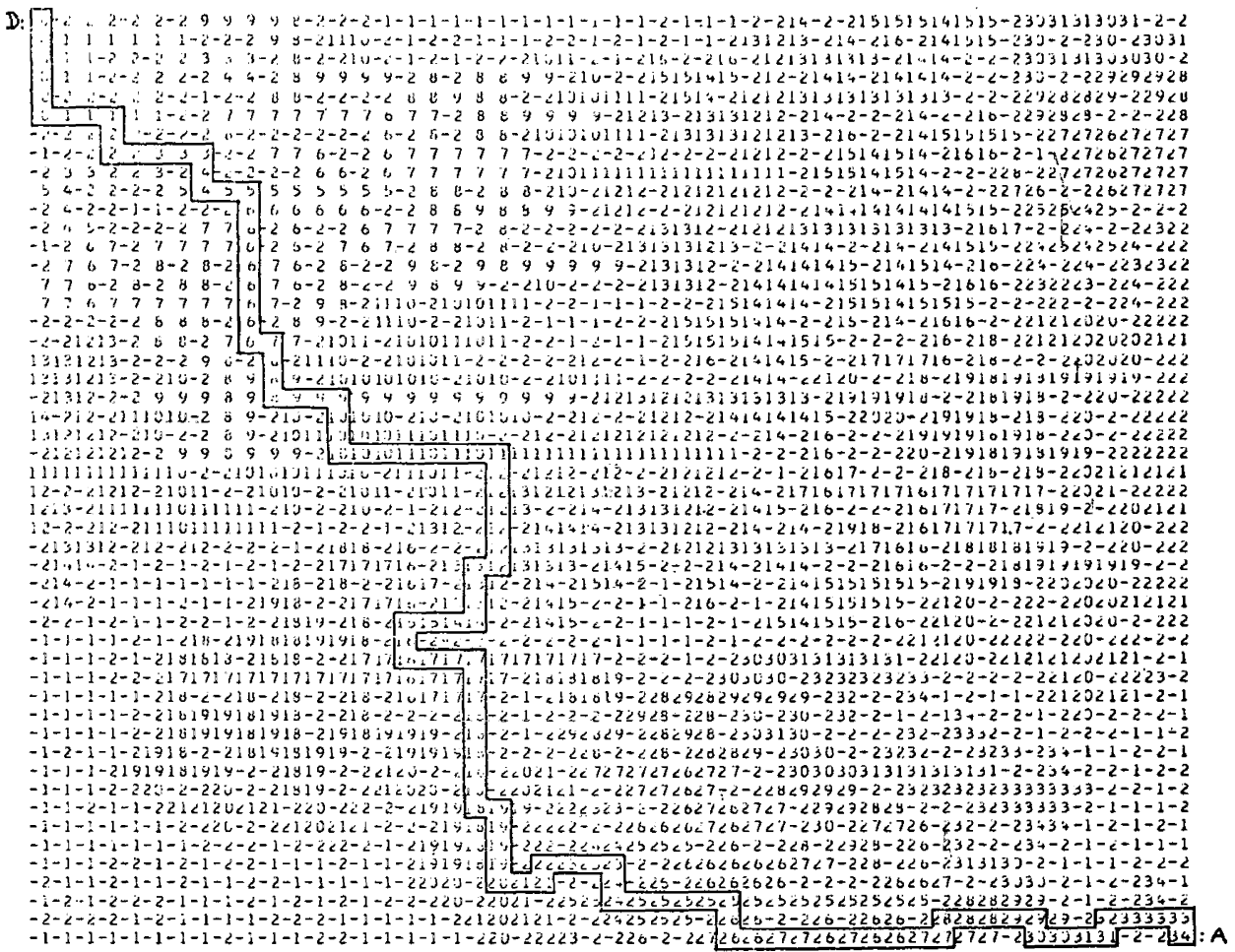

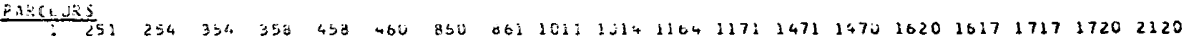

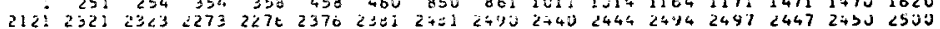

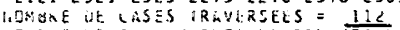
NOWBKE UE CHABEEREHTS DE DIKECTIUA
UUKEE

R.A.I.R.O. Recherche opérationnelle/Operations Research 
Algorithme multicritère : On reprend ici les deux exemples simples du paragraphe 6. Dans chaque niveau, chaque case $x$ est mémorisée sous la forme d'un entier avec 8 chiffres significatifs, les 4 de gauche indiquent le numéro de la case $x$, les 4 de droite indiquent le numéro de la case qui a permis d'atteindre $x$.

LABYRIATHE CE DEPARTIIOQ71: OEPART $=15$

LABYRINTHE, CE DEPARTI $7 \neq 61$

DEPART $=7$
ARRIVEE

\begin{tabular}{|c|c|c|c|c|c|c|c|}
\hline & & $\begin{array}{l}1 \\
77 \\
13 \\
19 \\
25 \\
31 \\
37\end{array}$ & $\begin{array}{r}2 \\
0 \\
14 \\
20 \\
26 \\
32 \\
38\end{array}$ & $\begin{array}{r}3 \\
9 \\
15 \\
4 \\
27 \\
0 \\
39\end{array}$ & $\begin{array}{ll}0 & \\
10 & 1 \\
16 & \\
22 & 2 \\
28 & \\
34 & 3 \\
40 & 4\end{array}$ & $\begin{array}{rr}5 & 0 \\
11 & 0 \\
0 & 18 \\
23 & 24 \\
0 & 20 \\
35 & 36 \\
41 & 0\end{array}$ & \\
\hline AIVEAL: & 0 & & .6000 & & & & \\
\hline AIVESL: & 1 & & .0007 & 1 & .0007 & & \\
\hline AIVEAU: & 2 & &. $\cos 3$ & & & & \\
\hline AIVEAL: & 3 & & .0019 & & & & \\
\hline AIVEAL: & 4 & & .0013 & & .0001 & $131 \cdot \operatorname{co} 25$ & \\
\hline AIVEAC: & 3 & & .0019 & & .0014 & 4.0002 & 37.0031 \\
\hline AIVEAC: & $t$ & & .0025 & & . vols & & \\
\hline AIVEAL: & 7 & & .0031 & & .0026 & & \\
\hline NIVEAL: & a & & .0015 & & .0003 & 38.0037 & $28 \cdot \mathrm{CO} 27$ \\
\hline AIVEAL: & 5 & & .1016 & & .0010 & 39.0038 & \\
\hline AIVEAL: & 10 & & .0039 & & & & \\
\hline NIVEAU: & 11 & & $.002 \mathrm{a}$ & & .0040 & & \\
\hline AIVEAL: & 12 & & .0022 & & .0010 & & \\
\hline AIVEAL: & 13 & & .0023 & & & & \\
\hline AIVEAL: & 14 & & .0034 & & .0041 & & \\
\hline AIVEAL: & 15 & & .0011 & & .0035 & & \\
\hline$\forall(c$ & & $V(A)=$ & $\begin{array}{l}15 \\
L A B Y R\end{array}$ & RINT & HE $F$ & IMAL & \\
\hline & & 1 & 4 & 5 & $-2 \quad 1$ & $15-2$ & \\
\hline & & u & -2 & 8 & 9 & 12 & \\
\hline & & $i$ & 4 & 5 & 0 & -2 & \\
\hline & & 2 & 5 & -2 & 9 & 12 & \\
\hline & & 3 & 6 & 7 & 8 & -2 & \\
\hline & & & 7 & -2 & 11 & 14 & \\
\hline & & s & 80 & 9 & io & ii & \\
\hline $\begin{array}{l}\text { NUM } \\
D E\end{array}$ & $\begin{array}{l}E \text { DE } \\
R E K\end{array}$ & $\begin{array}{l}\text { CASES } \\
\text { CAAS }\end{array}$ & $\begin{array}{l}\text { AUIC } \\
\qquad A R B\end{array}$ & $\begin{array}{l}\text { CRIS } \\
\text { BURE }\end{array}$ & $\begin{array}{l}\text { EES CA } \\
\text { SCENCE }\end{array}$ & $\begin{array}{l}\text { ENOILAIES } \\
\text { E: } \leqq 4\end{array}$ & \\
\hline A & & CASES & CANS & $5 \mathrm{~L}$ & ARBQRE & SECENCE: & 34 \\
\hline
\end{tabular}

\begin{tabular}{|c|c|c|c|c|c|c|c|c|}
\hline & & $\begin{array}{l}1 \\
8 \\
115 \\
22 \\
29 \\
36 \\
43 \\
50 \\
57 \\
64\end{array}$ & $\begin{array}{r}2 \\
0 \\
16 \\
0 \\
0 \\
5 \\
44 \\
51 \\
58 \\
65\end{array}$ & $\begin{array}{r}3 \\
0 \\
17 \\
24 \\
0 \\
0 \\
45 \\
52 \\
59 \\
66\end{array}$ & $\begin{array}{r}4 \\
0 \\
0 \\
25 \\
32 \\
0 \\
0 \\
0 \\
0 \\
67\end{array}$ & $\begin{array}{r}5 \\
0 \\
19 \\
0 \\
33 \\
0 \\
0 \\
0 \\
0 \\
03\end{array}$ & $\begin{array}{l}6 \\
13 \\
2 J \\
27 \\
34 \\
41 \\
49 \\
55 \\
5 \\
69\end{array}$ & $\begin{array}{r}7 \\
14 \\
21 \\
0 \\
0 \\
428 \\
49 \\
56 \\
63 \\
10\end{array}$ \\
\hline $\begin{array}{l}\text { AVEAL: } \\
\text { A IVEALL }\end{array}$ & $\begin{array}{l}0 \\
1\end{array}$ & & $\begin{array}{l}.0000 \\
.0015\end{array}$ & & 0013 & & 0015 & \\
\hline AIVEAL: & 2 & & co16 & & .0022 & 1 & . COOB & \\
\hline A IVEAL: & 3 & & .0029 & & & & & \\
\hline $\begin{array}{l}\text { NivEAL: } \\
\text { AIVEALL }\end{array}$ & $\overrightarrow{5}$ & &.$<036$ & & .0001 & & 10043 & \\
\hline $\begin{array}{l}\text { AIVEAL: } \\
\text { AI }\end{array}$ & $t^{3}$ & & .0002 & & .005 i & & 0004 & \\
\hline AIVEAL: & 9 & &. $\mathrm{CO}_{3} 3$ & & .0003 & & .0057 & \\
\hline AIVEAL: & $B$ & & .0024 & & .0050 & & .0044 & 5.000 \\
\hline AIVEAL: & $y$ & & .0057 & & .0051 & & . cuos & \\
\hline AIVEAL: & 10 & & .6064 & & .0050 & & . cu06 & \\
\hline AIVEAL: & 11 & & .0025 & & .0065 & & & \\
\hline AIVEAL: & 12 & & .0006 & & .0066 & & & \\
\hline RIVEAL: & 13 & & .0007 & & .0013 & 3 od. & .0067 & \\
\hline NIVEAL: & 14 & & .0032 & & . UU1 & +27 &.$c 020$ & 064.1068 \\
\hline AVEAL: & 15 & & .0033 & & .0027 & & .0064 & \\
\hline AIVEAL: & le & & .0020 & & .0034 & & & \\
\hline AIVEAL: & 17 & 48 & .6041 & & & & & \\
\hline AVEAL: & $1 a$ & is &.$c 034$ & & .0070 & & .0048 & \\
\hline RIVEAL: & 19 & & .0041 & & .0063 & & & \\
\hline \multirow{11}{*}{\multicolumn{2}{|c|}{$v([)=0$}} & $V(\theta)=$. & 15 & 5 & & & & \\
\hline & & 2 & 5 & 6 & 7 & 8 & $y$ & 10 \\
\hline & & 1 & $-\hat{2}$ & -2 & -2 & -2 & 12 & 13 \\
\hline & & 0 & 1 & 2 & -2 & 16 & 13 & 14 \\
\hline & & 1 & -2 & 5 & o & -2 & 14 & -2 \\
\hline & & 2 & -2 & -2 & 11 & 14 & 15 & -2 \\
\hline & & 3 & -2 & -2 & -2 & -2 & 16 & 19 \\
\hline & & 4 & 7 & 8 & -2 & -2 & 17 & 20 \\
\hline & & 5 & 8 & 9 & -2 & -2 & 18 & 19 \\
\hline & & 6 & 9 & 10 & -2 & -2 & -2 & 18 \\
\hline & & 9 & 10 & 11 & 12 & 13 & 14 & is \\
\hline \multirow{2}{*}{\multicolumn{2}{|c|}{$\begin{array}{l}\text { NUMGHE LE } \\
\text { A EGIREK } \\
\text { AUMGKE CE }\end{array}$}} & & & & & $\begin{array}{l}\text { Canol } \\
\text { Cẽ: }\end{array}$ & $\begin{array}{l}\text { IOATE } \\
\text { TU }\end{array}$ & \\
\hline & & & r. & & Ge & KESCE & EACE : & \\
\hline
\end{tabular}


Exemple $(70 \times 70):$

TAUX OE PRESENCE D OBSTACLES: .35

LAGYRINTHE DE DEPART

S1100C111010111100111111101101100011101101111111111100111011111111111 d 00100110011001110101101101111001100001111111011011110010111110001141

4 0111110110100111100100011011110101011110011116 $011101101111011111001011111110+1+12+1011111101111111111110100$ 0101000100110001110000111010161011111011041000111111101110010 H1111001111010111011110111011101 11110110101101101100111111111111011 10011C11110110111001111101110101 1110000001110011010001111111000 $0000001010101001011110011001 \mathrm{C01}$ 1001010100010101101000110101111 1101101011011111111010100101111
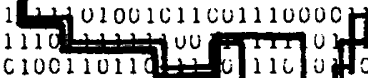
1100101101000111111110 101001101111101000110000011110011010 111010111110111100111100010100011111 111101011101110101111110111110011110 14+1011111100101101000 1110111100 0110121101011111101000111

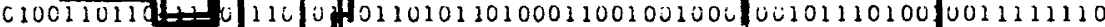
c11111110110100121. C10100C01000001C00U10001000111111011011100011110 1110111001411010111 $111011111010101111101110111010111010000000100111+110110011111110111$ C00000111100111101010110101101611100111011111111100111111111101110101 11011001111011101111011001111110100101100011010110001111111111111001 c10111110101110100111101010100111011100011100101014+1400 v0101011 11110110111100010100000001101161100111111111000160001111411111111101 11001111100100101111111001111110010110101010110111010100 ul 110010001 10101111110100111001100111000061011011111111101110011001110110001001 001111001001111100111010111001010101010111010011111110011 U1 101110010 C10111C1010111101001111000001110101111100010100101111C1HA11 001000000 100110101110110011100111001001111011011111010101000001011001 lL0U1U1U1 $111111110110101101001111110111111001100100100110110000+11101111111111$ 010000111110001011101111111011110010110100010001110110001001111111100 110010000011110101110111000111100111011110110101111111111111111100011 c10111100011116101111101011011111111111000001001011110 00110 011110010 010010111000110110000101110110101111101110110001111110 $1000111010001011110111111111110111100111110110110011110_{11100110011110}$ 111010110101010111010101011011101001111111110010010000140111110111100 $1 C 11111111101110010100101101111101100011111011010101110$ 11010111114011 11111111111110111111011001101111111001011111011111100 d1111111010111 611011010111001011110101001011001110111101200011101110010111101101110 c01100111101111100101001100110010011101100110101101165010100110111010 c0101000111011111011110110111011010110101101011110110c 111111101001011 1101110110111001006001011001111101101011011101111001109010111111100110 C01111110000101010111110001001001000011100110001111014011011100011101 111111101001010111100110111011101000111110001110111110100100011000010 CC110161111111110111116011111111100010011111101110100) 1000111161111100 10110110101111011011111111111110111101101100100001111 th0100101001001 110101111100101100111111111110001111111111101011001116 h 1100101111110 c1100111101101000100111110011101111100100001101110111101 0001111101101 01101101111111101111101011110111101111001010110110110010 0111000011001 C01010C1011011011001110011101111100010111010011111011110\% FT 101011011101110011111001111000001110110111110111111011100101110011101 11111011011101110101111101011110011011011111111111010110110 U1 1110100 C0101111111111111111110111011011001111110110110001101110111111 1000000 11010010001101101011011101111161000111110000101101100011101 th 11110 $111111111110000110111110011101000110001101111111111111100110110^{1} 01010$ C0101101111111111001111111101111111100001111111111101101110001 1 10001 01111111010011100100110010111101011101111111000001010120101111 t2010 111111001001011110111111101111010011111110011100111101010001110011010 11010110000111101011001110111111011100111101101011100110100011110111 0110011110011010111001110010010110100001001100001111111111101100411 11010111011.1011101100110111110610011011010111110116111010110101000110 111001000111001001011111111010111101011011111010110101001111111100110 11010111000111001110010010001111011101101100010101010111101010011010 0101000000101120011100110011000101111111111111011111110001111011110 U1 11010111101110101111111001111100001011011101110011011110111000010004 11001010111001111000011011110111101100111111101011001110101010010110 11101010.101111100111111010101101110111111011010110010111000010101001 1000101110111101111111111110111101101100010111100001111101001011011010 C01100C1001101111010001011011111111111001011101111011111111010000111 C00000010101111111011001111111C0010011101101010010011101111111001010 11111111111010011010100010011111101111101101001011101101111111000111 . C1111100101111001111011011011111010011000101010011110110100111011011 C11010111110001011110100100111100001010101111111111001010010001101119

Les résultats des deux algorithmes sur cet exemple sont donnés page suivante, où les cases sont numérotées de 1 à 4900 . 


\section{Multicritère :}

IAUX DE PRESENCE D DHSTACLES:.35

$(v)=0 \quad V(A)=268$

LBYRIIUTHE EIMAL

NCMELE CE CASES AUTORISEES CAMDIJATES

A ENTRER WAISS L ARBURESCEHCE: 51953

PERCCLES: 4YCL $48 \times 4 \quad 402947594634461945494479440443394330420841984197412740573987391730673717$

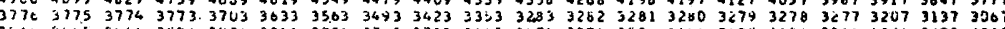

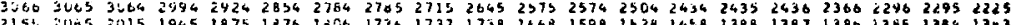
215130052015194518751876 1006 1736173717381668159823281438138813871380138513961303

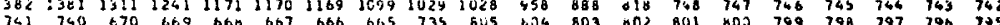

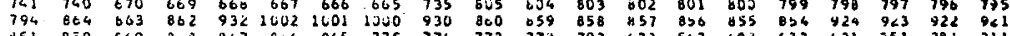
$\begin{array}{lllllllllllllllllll}d 51 & 850 & 649 & 8 \times 8 & 847 & 806 & 845,775 & 776 & 773 & 772 & 702 & 632 & 562 & 692 & 422 & 421 & 351 & 281 & 211\end{array}$ OMEKE DE CHANGERENTS UE OLRTSTHUY: 52 CUREE:

\section{Hiérarchie :}

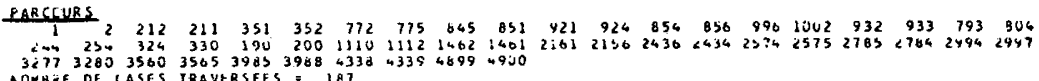
$327732803560356539833988433 d 433948994900$ AOPBKE DE CASES TRAVERSES 


\section{Programmes :}

HIERARCHIE:3

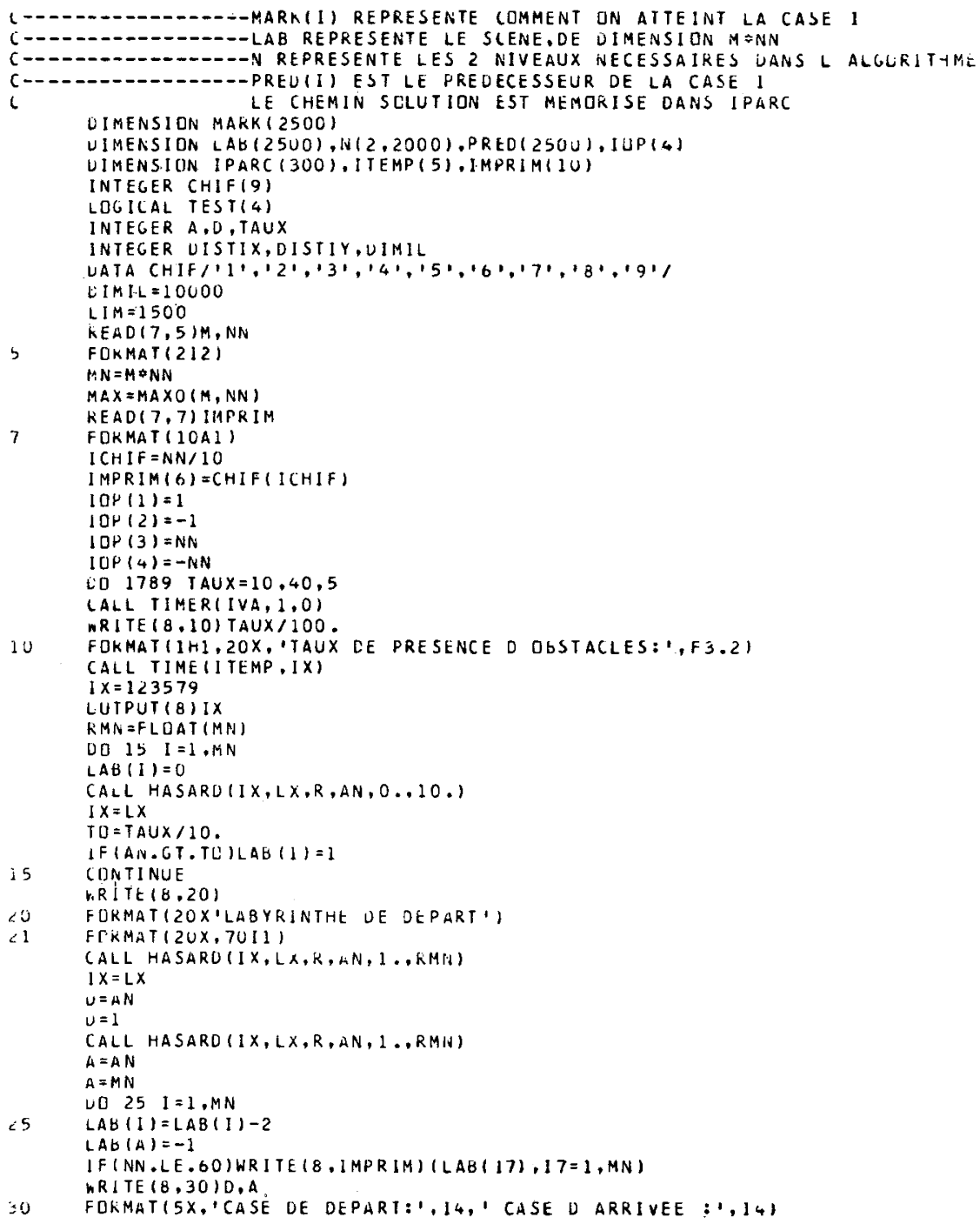

R.A.I.R.O. Recherche opérationnelle/Operations Research 


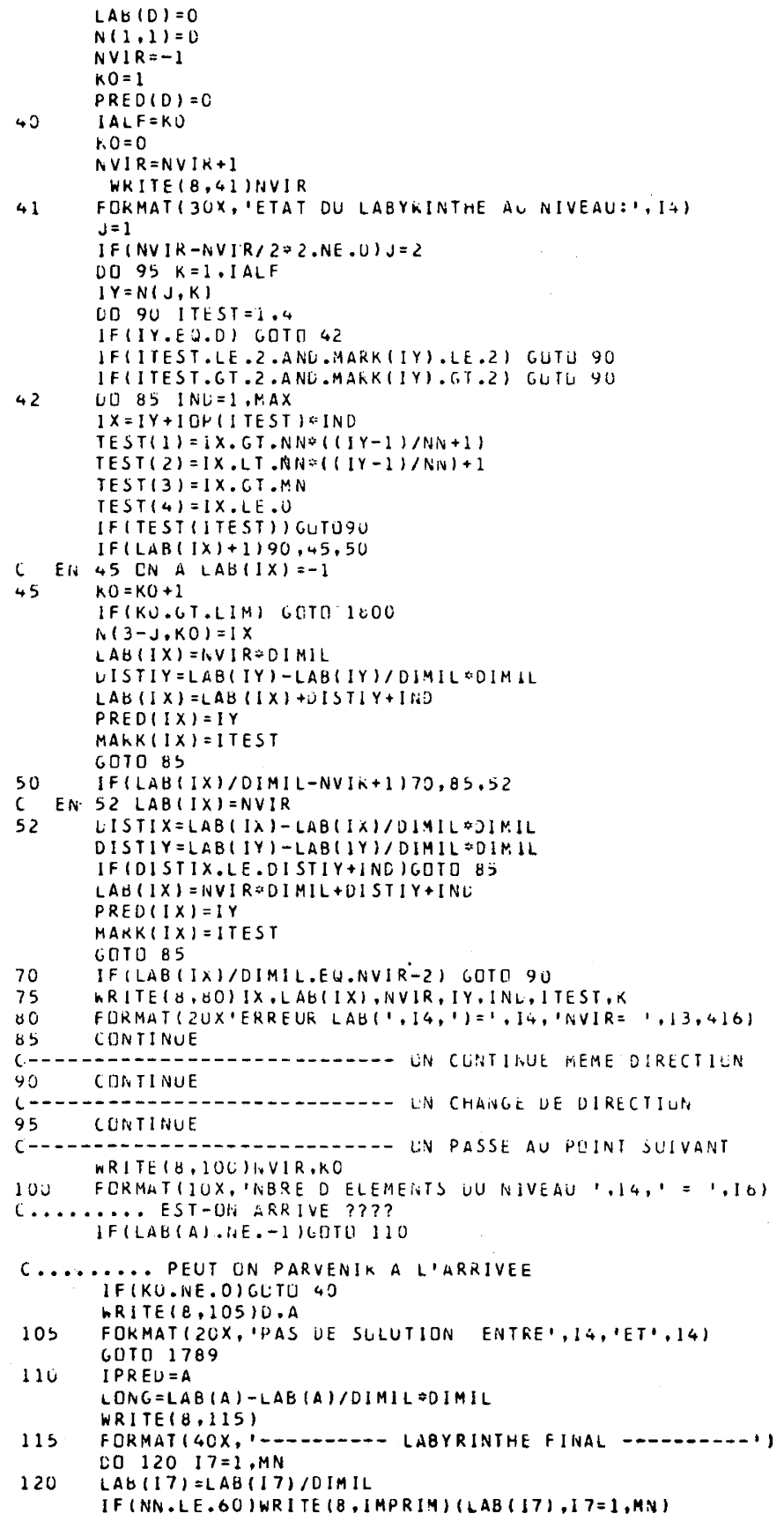




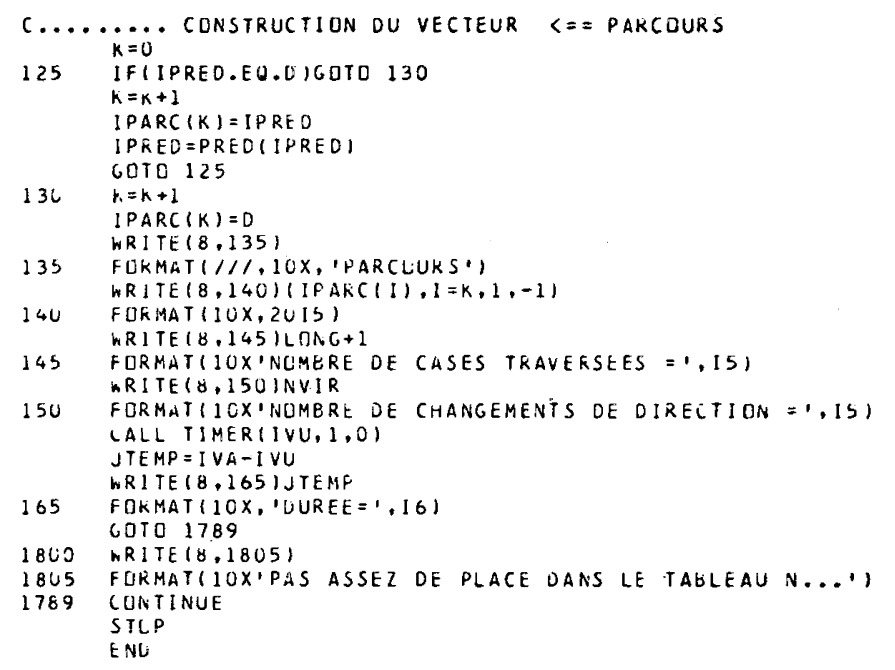

\section{BIBLIOGRAPHIE}

1. M. Delannoy, M. Leror et M. Bourton, Recherche d'un chemin optimal dans une scène planifiée, $2^{\mathrm{e}}$ congrès A.F.C.E.T.-I.R.I.A.; Reconnaissance des formes et intelligence artificielle, septembre 1979, Toulouse.

2. Gondran et Minoux, Graphes et algorithmes, Eyrolles, 1979.

3. HART, Nilsson et RaphaEl, A formal Basis for the Heuristic Determination of Minimum Cost Paths, I.E.E.E., vol. SSC-4, n² 2, 1968.

4. Giralt, Sobek et Chatila, A Multi-Level Planning and Navigation System for a Mobil Robot: a First Approach to Hilare, Sixth International Joint Conference on Artificial Intelligence, 20-24/8/1979, Tokyo.

5. Thompson, The Navigation system of the J.P.L. Robot, Proceedings of the I.J.C.A.I., août 1977 , p. $749-757$.

6. Bitner et REINGOLD, Backtrack programming techniques, Comm. of the A.C.M., vol. $18, \mathrm{n}^{\circ} 11$, novembre 1975 , p. 651-656.

7. J. R. CAYrol, Conception de la simulation d'un robot, Thèse $3^{e}$ cycle, 1978, Toulouse.

8. PoHL, Heuristic Search Viewed as Path Finding in a Graph, Artificial Intelligence, vol. 1, 1970, p. 193-204.

9. Doran et Michie, Experiements With the Graph Traverser Program, Proc. Roy. Soc., vol. A, no 294, 1966, p. 235.

10. M. Shimura, Heuristic Problem Solving by Tree Search, Systems Computers Controls, vol. 8, $\mathrm{n}^{\circ} 4,1977$.

11. D. E. KNUTH, The Art of Computer Programming, vol. 1. Fundamentals Algorithms, Addison-Wesley, 1973.

12. MeYer et BAUdoIN, Méthodes de programmation, Eyrolles, 1978.

R.A.I.R.O. Recherche opérationnelle/Operations Research 